\title{
The child with chronic cough: when does double-channel pH monitoring rule out gastroesophageal reflux?
}

\author{
Richard Kitz $^{1 *}$, Peter Ahrens ${ }^{2}$, Olaf Eickmeier ${ }^{1}$, Hansjosef Boehles ${ }^{1}$, Markus A. Rose $^{1}$ \\ ${ }^{1}$ Children’s Hospital, Goethe University, Frankfurt, Germany; \\ ${ }^{2}$ Prinzessin Margaret Children’s Hospital, Darmstadt, Germany. \\ Email: *R.Kitz@ckhf.de
}

Received 13 May 2011; revised 3 July 2011; accepted 19 July 2011.

\begin{abstract}
Background: gastroesophageal reflux (GER) plays a major role in the pathogenesis of pediatric chronic airway disease. Esophageal pH-monitoring (epHM) is the diagnostic gold standard for acid GER. To date, there are no cut-off values for chronically coughing children ruling out relevant GER. Methods: 24-hour, double-channel epHM was performed in 549 children (3 months to 16 years old) with chronic pulmonary disease. We stratified according to age as follows: $\leq$ 1.5 years, $>1.5$ - 4 years, $>4-8$ years, $>8<12$ years and $>12$ - 16 years. Following parameters were calculated for both channels: total number of reflux episodes, number of reflux episodes $>5$ minutes, duration of the longest reflux episode, and reflux index. Results: according to the above given age classification, the median number of reflux episodes in the lower esophagus was 31, 27, 32, 34, and 42 and for the upper esophagus $20,13,15,14$, and 11 respectively. The median reflux index at the distal esophagus was $2.55,2.1,2.3,2.15$, and 1.9; at the upper esophagus it was $1.4,1.0,1.1,0.9$, and 0.6 respectively. Conclusions: our data contribute useful support to the evaluation of pediatric airway disease. We provide reference values for decisions in the exploration of children with airway disorders and suspected GER.
\end{abstract}

Keywords: Gastroesophageal Reflux; Pediatric Airway Diseases; pH-Monitoring; Chronic Aspiration

\section{INTRODUCTION}

Numerous studies in chronic airway disease emphasize the etiological role of gastroesophageal reflux (GER), some of them also proving a significant epidemiological correlation. The prevalence of respiratory symptoms among patients with GER is high. Monitoring of esophageal $\mathrm{pH}$ (epHM) is considered to be the gold stan- dard in detecting classic GER. The North American Society for Pediatric Gastroenterology and Nutrition recommends performing epHM in patients with persistent asthma, even in the absence of symptoms of GER, and recommends medical treatment if epHM demonstrates an increased frequency or duration of esophageal acid exposure [1]. In asthmatic children with GER, antacid treatment resulted in a significant reduction in asthma medication [2].

Astonishingly, data upon normal values of acid exposure of the esophagus in children with unspecific pulmonary symptoms, measured by $\mathrm{pH}$-monitoring, are scarce. There are only three studies providing data on multiple sites measuring in the esophagus: Bagucka et al. provided double-channel $\mathrm{pH}$ monitoring normal ranges of gastroesophageal reflux parameters in the upper esophagus in 200 infants aged 0.5 - 17 months, referred with suspected GER disease [3]. Sondheimer [4] measured $\mathrm{pH}$ at three levels in the esophagus in only 11 children without pathological GER and in 14 with pathological GER. In an older study [5] on 27 healthy children and adolescents (9.3 - 17.3 years old, mean 13.1) by Gustafsson in 1988, values for the upper probe, $15 \mathrm{~cm}$ above the cardia, were given as follows: RI $0.6 \%$, number of reflux episodes in 19/24 h; the duration of longest reflux episode being 3.3 minutes.

To date, there are no reference values available for the upper esophagus in children with unspecific pulmonary symptoms, but without typical gastrointestinal symptoms of GER (e.g., vomiting). This is the first study to validate the standard method in detecting GER in these children from infancy to adolescence.

\section{MATERIALS AND METHODS}

Patients. At our institution, children undergo epHM in search of evidence of GER when presenting chronic cough throughout the year, recurrent wheezing, or recurrent bronchitis/pneumonia. Among those and over a period of six years, 549 children (aged 3 months to 16 
years) had a normal epHM according to ESPGHANprotocol criteria (reflux index at the distal esophagus < 5\%; see below) and were included into this study [6]. Exclusion criteria were other serious chronic diseases (e.g., cystic fibrosis, perennial allergic disease, or food allergy), neurological disorders with the risk of dysphagia, immunodeficiency, malformations of the tracheobronchial tree, or chronic foreign body aspiration. At the time of the study, none of the patients was on acid suppression therapy or on theophylline antiasthmatic treatment.

Examinations were performed on a walk-in basis. Informed consent was obtained from the patients or the patients' guardians ahead of the examination. Guardians were asked to withhold their children's normal diet and allow customary sleeping positions throughout the 24hours period. Ethical approval was not obtained because of the study's retrospective design. For ethical reasons, we renounced examining a healthy control group.

Continuous epHM was performed according to the ESPGHAN standardized protocol. A monocrystalline antimony double-channel pH probe (Medtronic Synectics Medical, Sweden) was calibrated in buffers of $\mathrm{pH}$ 7.01 and $\mathrm{pH} 1.07$ and then placed transnasaly into the esophagus. The distance of the two $\mathrm{pH}$ sensors on the probe was adjusted according to the body height of the patient, using our previously published formula [7]. After adjusting the probe for an optimal position within the esophagus, with the use of a control chest x-ray, with the upper probe position of the sensors between the clavicles. The lower probe position was set in the distal esophagus. Therefore we used three different distances between the sensors: $5 \mathrm{~cm}$ in patients with a body height of $\leq 80 \mathrm{~cm}$, $10 \mathrm{~cm}$ in patients with a body height of $\leq 120 \mathrm{~cm}$, and 15 $\mathrm{cm}$ in patients with a body height of $>120 \mathrm{~cm}$. This facilitates interindividual comparability for epHM. The probes were then connected to a portable digital recorder (Digitrapper MK III, Synectics Medical AB, Sweden). Patients and their guardians were then asked to keep a diary for the next 24 hours. This diary included data on the time and kind of consumed meals, beverages and drugs as well as posture. After 24 hours, data were analyzed by the software "Esophogram ${ }^{\circledR}$ " (Synectics Medical AB, Sweden). Reflux episodes were defined as a decrease of esophageal ph below 4 for longer than five seconds followed by an increase of $\mathrm{pH}$ for minimum of $\mathrm{pH} 4.5$, thus avoiding oscillating phenomena. This enabled us to detect the number of reflux episodes at each sensor in the past 24 hours, the longest reflux episode and the number of long lasting reflux episodes ( $>5$ minutes). The summarized time of all reflux episodes divided by the total recording time (in general over a period of 24 hours) is the "reflux index" (RI).
Statistical analysis:

Statistical analysis was done with the software package SPSS for Windows ${ }^{\circledR}$ version 11.0 (SPSS Inc., Chicago, Illinois/USA). Data of the study population were tested for Gaussian distribution, however, KolmogorovSmirnoff's delta showed no normal distribution. Thus, we decided to choose nonparametrical statistic testing. Medians and interquartile ranges (IQR) between the $25 \%$ and $75 \%$ percentile, provide relevant descriptive parameters. A Kruskal-Wallis analysis was performed to compare epHM parameters of all age groups. In order to facilitate a comparison with literature data, we additionally provide mean values as well as standard deviations.

\section{RESULTS}

Among 549 patients with normal lower reflux index (RI) values (<5\%), 126 were younger than 18 months, 148 were 18 months to 4 years old, 195 were 4 to 8 years old, 47 were 8 to 12 years old, and 33 were 12 to 16 years of age.

Results for our four epHM parameters, studied in patients with distal RI $<5 \%$, are listed in Table 1. Overall, there was less reflux in the upper esophagus than in the lower esophagus. Infants between 3 to 18 months of age had the highest reflux activity of all examined age groups. As expected, physiological reflux diminishes as maturation of the cardia proceeds. In all age groups, reflux episodes lasting longer than five minutes are scarce and longer reflux episodes tended to occur in older children, while the total number of reflux episodes was higher in younger children.

When comparing data of the age groups older than 18 months, we only found a trend toward slight reflux activity depending on age. No statistical significant differences for the epHM parameters could be found in the Kruskal-Wallis analysis. Therefore, we decided to pool children between the ages of 18 months to 16 years as one group (Table 2). This was also true for children with higher lower esophageal RIs (data not shown).

In infants younger than 18 months of age and normal RIs in the lower esophagus, the median number of reflux episodes in the upper esophagus was 21 (IQR 10 - 31) over a 24-hour period. While the longest reflux episode lasted 4 min. (IQR 2.0 - 8.2 minutes), the median number of episodes lasting more than 5 minutes was 0 (IQR 0 - 1) over a 24-hour period. The resulting reflux index (RI) in the upper esophagus was 1.4 (IQR 0.5 - 2.5). Children between the ages of 18 months to 16 years had a total number of 14 (IQR 7 - 24) reflux episodes in the upper esophagus. Their longest episode lasted 4 minutes (IQR 2 - $8 \mathrm{~min}$ ). The median number of reflux episodes longer than 5 minutes was 0 (IQR 0 - 1). The resulting reflux index RI was 1.0 (IQR 0.4 - 2) in this age group. 
Table 1. Upper and lower esophageal $\mathrm{pH}$ monitoring parameters in patients with normal distal reflux index (RI) values $(<5 \%)$ stratified for patient age.

\begin{tabular}{|c|c|c|c|c|c|c|c|c|c|c|}
\hline Age (years) & \multicolumn{2}{|c|}{$\begin{array}{c}3-<18 \text { months } \\
n=126\end{array}$} & \multicolumn{2}{|c|}{$\begin{array}{c}1.5-<4 \text { years } \\
n=148\end{array}$} & \multicolumn{2}{|c|}{$\begin{array}{c}4-<8 \text { years } \\
n=195\end{array}$} & \multicolumn{2}{|c|}{$\begin{array}{c}8-<12 \text { years } \\
n=47\end{array}$} & \multicolumn{2}{|c|}{$\begin{array}{c}12-16 \text { years } \\
n=33\end{array}$} \\
\hline Mean & \multicolumn{2}{|c|}{0.87} & \multicolumn{2}{|c|}{2.60} & \multicolumn{2}{|c|}{5.51} & \multicolumn{2}{|c|}{9.46} & \multicolumn{2}{|c|}{13.91} \\
\hline SD & \multicolumn{2}{|c|}{0.34} & \multicolumn{2}{|c|}{0.74} & \multicolumn{2}{|c|}{1.10} & \multicolumn{2}{|c|}{1.10} & \multicolumn{2}{|c|}{1.14} \\
\hline $25 \%$ quartile & \multicolumn{2}{|c|}{0.59} & \multicolumn{2}{|c|}{1.86} & \multicolumn{2}{|c|}{4.55} & \multicolumn{2}{|c|}{8.59} & \multicolumn{2}{|c|}{13.04} \\
\hline Median & \multicolumn{2}{|c|}{0.85} & \multicolumn{2}{|c|}{2.46} & \multicolumn{2}{|c|}{5.33} & \multicolumn{2}{|c|}{9.16} & & \\
\hline SEM & & & & & & & & & & \\
\hline $75 \%$ quartile & & & & & & & & & & \\
\hline & & & & f reflus & sodes & & & & & \\
\hline & upper & lower & upper & lower & upper & lower & upper & lower & upper & upper \\
\hline Mean & 21.80 & 31.96 & 17.20 & 29.75 & 16.93 & 35.50 & 19.53 & 47.48 & 15.30 & 44.82 \\
\hline SD & 14.90 & 18.04 & 13.56 & 19.31 & 12.75 & 26.27 & 19.78 & 64.88 & 14.03 & 24.65 \\
\hline $25 \%$ quartile & 10.00 & 21.00 & 7.00 & 17.25 & 7.00 & 18.00 & 8.00 & 16.00 & 6.00 & 27.50 \\
\hline Median & 20.50 & 31.00 & 13.00 & 27.00 & 15.00 & 32.00 & 14.00 & 34.00 & 11.00 & 42.00 \\
\hline SEM & 1.33 & 1.61 & 1.11 & 1.59 & 0.91 & 1.88 & 2.89 & 9.36 & 2.44 & 4.29 \\
\hline $75 \%$ quartile & 31.00 & 40.25 & 26.75 & 39.00 & 23.00 & 47.00 & 26.00 & 53.75 & 23.50 & 55.50 \\
\hline$p$ (dist./prox.) & & & & & & & & & & \\
\hline & & & No. $r$ & -episod & 5 minu & & & & & \\
\hline Mean & 0.70 & 0.85 & 0.95 & 1.00 & 0.76 & 0.99 & 0.55 & 0.77 & 0.81 & 1.15 \\
\hline SD & 1.20 & 1.07 & 1.49 & 1.18 & 1.07 & 1.02 & 1.33 & 0.90 & 1.65 & 1.32 \\
\hline $25 \%$ quartile & 0.00 & 0.00 & 0.00 & 0.00 & 0.00 & 0.00 & 0.00 & 0.00 & 0.00 & 0.00 \\
\hline Median & 0.00 & 0.00 & 0.00 & 1.00 & 0.00 & 1.00 & 0.00 & 0.50 & 0.00 & 1.00 \\
\hline SEM & 0.10 & 0.09 & 0.12 & 0.10 & 0.08 & 0.07 & 0.19 & 0.13 & 0.29 & 0.23 \\
\hline $75 \%$ quartile & 1.00 & 1.00 & 1.00 & 1.75 & 1.00 & 2.00 & 1.00 & 1.00 & 1.00 & 2.00 \\
\hline$p$ (dist./prox.) & & & & & & & & & & \\
\hline & & & & est refl & pisode & & & & & \\
\hline Mean & 6.20 & 7.18 & 6.88 & 7.43 & 8.10 & 8.18 & 6.42 & 7.37 & 12.39 & 8.76 \\
\hline SD & 6.50 & 5.99 & 7.80 & 6.35 & 11.21 & 7.10 & 9.67 & 6.78 & 23.41 & 8.42 \\
\hline $25 \%$ quartile & 2.00 & 3.00 & 2.00 & 3.00 & 2.00 & 3.00 & 1.00 & 3.00 & 1.00 & 2.50 \\
\hline Median & 4.00 & 5.00 & 5.00 & 6.00 & 5.00 & 6.00 & 3.00 & 4.50 & 3.00 & 6.00 \\
\hline SEM & 0.58 & 0.54 & 0.64 & 0.52 & 0.80 & 0.51 & 1.41 & 0.98 & 4.08 & 1.47 \\
\hline $75 \%$ quartile & 8.20 & 11.00 & 8.00 & 10.00 & 9.00 & 11.00 & 7.00 & 10.50 & 13.50 & 11.00 \\
\hline$p$ (dist./prox.) & & & & & & & & & & \\
\hline & & & & flux-In & $(\%)$ & & & & & \\
\hline Mean & 1.70 & 2.43 & 1.64 & 2.20 & 1.51 & 2.31 & 1.37 & 2.19 & 1.96 & 2.44 \\
\hline SD & 1.60 & 1.43 & 1.97 & 1.37 & 1.59 & 1.33 & 1.93 & 1.41 & 3.46 & 1.50 \\
\hline $25 \%$ quartile & 0.50 & 1.20 & 0.40 & 1.00 & 0.50 & 1.20 & 0.30 & 1.12 & 0.20 & 1.15 \\
\hline Median & 1.40 & 2.55 & 1.00 & 2.10 & 1.10 & 2.30 & 0.90 & 2.15 & 0.60 & 1.90 \\
\hline SEM & 0.14 & 0.13 & 0.16 & 0.11 & 0.11 & 0.09 & 0.28 & 0.20 & 0.60 & 0.26 \\
\hline $75 \%$ quartile & 2.50 & 3.52 & 2.17 & 3.30 & 2.00 & 3.30 & 1.60 & 3.40 & 2.45 & 4.00 \\
\hline$p$ (dist./prox.) & & & & & & & & & & \\
\hline
\end{tabular}

SEM = standard error of means; $\mathrm{SD}=$ standard deviation. 
Table 2. Upper and lower esophageal $\mathrm{pH}$ monitoring parameters in patients with normal distal reflux index (RI) values (<5\%). Comparison of infants and children up to 16 years of age.

\begin{tabular}{|c|c|c|c|c|}
\hline Age (years) & \multicolumn{2}{|c|}{$\begin{array}{c}\text { Infants }(3-<18 \text { months }) \\
n=126\end{array}$} & \multicolumn{2}{|c|}{$\begin{array}{l}\text { Children (18 months - } 16 \text { years) } \\
\qquad n=423\end{array}$} \\
\hline Mean & \multicolumn{2}{|c|}{0.87} & \multicolumn{2}{|c|}{5.62} \\
\hline SD & \multicolumn{2}{|c|}{0.34} & \multicolumn{2}{|c|}{3.37} \\
\hline $25 \%$ quartile & \multicolumn{2}{|c|}{0.59} & \multicolumn{2}{|c|}{3.19} \\
\hline Median & \multicolumn{2}{|c|}{0.85} & \multicolumn{2}{|c|}{4.89} \\
\hline SEM & \multicolumn{2}{|c|}{0.03} & \multicolumn{2}{|c|}{0.16} \\
\hline 75\% quartile & \multicolumn{2}{|c|}{1.22} & \multicolumn{2}{|c|}{7.18} \\
\hline \multicolumn{5}{|c|}{ No. of reflux episodes } \\
\hline & upper & lower & upper & lower \\
\hline Mean & 21.80 & 31.96 & 17.19 & 35.57 \\
\hline SD & 14.90 & 18.04 & 14.05 & 31.53 \\
\hline $25 \%$ quartile & 10.00 & 21.00 & 7.00 & 18.00 \\
\hline Median & 20.50 & 31.00 & 14.00 & 31.00 \\
\hline SEM & 1.33 & 1.61 & 0.68 & 1.53 \\
\hline $75 \%$ quartile & 31.00 & 40.25 & 24.00 & 45.00 \\
\hline \multicolumn{5}{|c|}{ No. of reflux episodes lasting longer than 5 minutes } \\
\hline mean & 0.70 & 0.85 & 0.81 & 0.98 \\
\hline SD & 1.20 & 1.07 & 1.31 & 1.09 \\
\hline $25 \%$ quartile & 0.00 & 0.00 & 0.00 & 0.00 \\
\hline median & 0.00 & 0.00 & 0.00 & 1.00 \\
\hline SEM & 0.10 & 0.09 & 0.06 & 0.05 \\
\hline $75 \%$ quartile & 1.00 & 1.00 & 1.00 & 2.00 \\
\hline \multicolumn{5}{|c|}{ Longest reflux episode (minutes) } \\
\hline mean & 6.20 & 7.18 & 7.82 & 7.87 \\
\hline $\mathrm{SD}$ & 6.50 & 5.99 & 11.53 & 6.91 \\
\hline $25 \%$ quartile & 2.00 & 3.00 & 2.00 & 3.00 \\
\hline median & 4.00 & 5.00 & 4.00 & 6.00 \\
\hline SEM & 0.58 & 0.54 & 0.56 & 0.34 \\
\hline $75 \%$ quartile & 8.20 & 11.00 & 8.00 & 11.00 \\
\hline \multicolumn{5}{|c|}{ Reflux-Index } \\
\hline mean & 1.70 & 2.43 & 1.57 & 2.27 \\
\hline SD & 1.60 & 1.43 & 1.96 & 1.37 \\
\hline $25 \%$ quartile & 0.50 & 1.20 & 0.40 & 1.10 \\
\hline median & 1.40 & 2.55 & 1.00 & 2.20 \\
\hline SEM & 0.14 & 0.13 & 0.10 & 0.07 \\
\hline $75 \%$ quartile & 2.50 & 3.52 & 2.00 & 3.37 \\
\hline
\end{tabular}

SEM = standard error of means; SD = standard deviation.

Statistical significant differences for epHM parameters were found for the total number of reflux episodes $(p<0.01)$ and the RI $(p<0.01)$, but not for the longest episode and the number of episodes lasting longer than 5 minutes.

\section{DISCUSSION}

When GER is considered as a cause of chronic airway disease, 2-channel epHM is the diagnostic tool of choice to confirm diagnosis. In clinical practice, GER as the cause of coughing may not be readily apparent, or cough may be the sole presenting symptom of GER. Each level of the airways can be affected by GER [8]. Cough can result from laryngeal irritation, from esophageal vagal induced bronchoconstriction, or from pulmonary microaspiration. Similar mechanisms have been postulated to explain chronic GER-related respiratory disease. None- theless, most of these children do not present gastrointestinal complaints [9]. It is therefore obvious that recording of esophageal $\mathrm{pH}$ may detect acid-induced bronchoconstriction (asthma) and measuring the proximal esophageal acid exposure may help to better detect microaspiration.

In adults, Harding identified 18\% of 1983 patients with pathologic $\mathrm{pH}$-recordings with self-reported asthma according to ATS criteria [10]. The absence of clinical reflux symptoms in these asthmatics with GER (known as "silent reflux") was frequent (18\%). The German ProGERD study in 6215 adult patients with GER found prevalences for chronic extraoesophageal disorders in chronic cough of $32.8 \%$, in laryngeal disorders of $4.8 \%$, and in asthma of 13\% [11]. An American case-control study compared 1980 children aged 2 - 18 years with GERD and 7920 controls without GER, showing a sev- 
eral-fold increased risk for sinusitis, asthma, pneumonia, and bronchiectasis [12]. Others found a high incidence of GER in asthmatic pediatric populations [13,14]. Values of esophageal $\mathrm{pH}(\mathrm{epH})$ are the highest in a subgroup of infants with chronic respiratory disorders undergoing 24-hour pH-monitoring [15]. Studies dealing with the impact of epHM on respiratory diseases should reflect these theories and normal values of acid exposure are of great importance. This is even more important, since overestimation of GER as a cause of asthma symptoms may lead to nonjustified therapy of GER as asthma treatment [16].

Reference values are mandatory to distinguish between physiological and pathologic reflux activity. Detecting the reflux in the upper esophagus may be of special interest in children with extraesophageal disorders. While older studies propose the $95^{\text {th }}$ percentile as the cut-off value [17], more recent publications favor the SEM as range of normal values [3]. Our study provides both limits in order to give a solid base for data interpretation. In order to avoid an over-treatment, we recommend the conservative approach of taking values beyond the $75^{\text {th }}$ interquartile range as an indication for an antiacid treatment.

Bagucka et al. [3] published normal data for the upper esophagus in children initially referred for exclusion of suspected GER. According to ESPGHAN-recommendations, they stratified their study group depending on the RI in the lower esophagus. While their values encompassed infants younger than 17 months of age, we now extended the age range up to 16 year-old adolescents. When comparing the data, we found consistencies in the total number of reflux episodes and the absence of episodes lasting longer than five minutes. Discrepancies were found for the RI $(0.5 \%$ vs $1.2 \%)$ in the present study. These findings could be explained by the different mean ages of the groups $<1.5$ years. Bagucka et al. studied infants as young as 0.5 months old, whereas in our study no child was younger than 3 months. Their median age was 3.0 months, whereas our children had a median age of 10.2 months. Taking into account the more buffered stomach content of milk-fed children, less amount of reflux can be detected by measuring only $\mathrm{pH}$-condition [18]. In other words: the longer the $\mathrm{pH}$ in the stomach is below 4 , the more reflux episodes can be detected in the esophagus. More precisely, simultaneously performed intraluminal impedance measurement of the esophagus can detect bolus movements, thus broadening our diagnostic spectrum as to detect nonacid reflux activity in the future [19,20].

Problems in the application of reference values may occur due to the type of recording device and electrodes. At present, mostly antimony electrodes are used. They are less accurate than glass electrodes, however, they provide multi-channel recording on different levels in the esophagus and are easier to handle as well as to place into the esophagus.

\section{CONCLUSIONS}

The present study provides reference values of proximal esophageal $\mathrm{pH}$-monitoring from infancy to adolescence. The inter-quartile ranges appear as suitable threshold levels when anti-acid treatment for the control of reflux-associated airway disease is considered.

\section{REFERENCES}

[1] Rudolph, C.D., Mazur, L.J., Liptak, G.S., Baker, R.D., Boyle, J.T., et al. (2001) Guidelines for evaluation and treatment of gastroesophageal reflux in infants and children: recommendations of the North American Society for Pediatric Gastroenterology and Nutrition. Journal of Pediatric Gastroenterology and Nutrition, 32, S1-S31. doi:10.1097/00005176-200100002-00001

[2] Khoshoo, V., Le, T., Haydel, R.M., Landry, L. and Nelson, C. (2003) Role of gastroesophageal reflux in older children with persistent asthma. Chest, 123, 1008-1013. doi:10.1378/chest.123.4.1008

[3] Bagucka, B., Badriul, H., Vandemaele, K., Troch, E. and Vandenplas, Y. (2000) Normal ranges of continuous $\mathrm{pH}-$ monitoring in the proximal esophagus. Journal of Pediatric Gastroenterology and Nutrition, 31, 244-247. doi:10.1097/00005176-200009000-00008

[4] Sondheimer, J.M. and Haase, G.M. (1988) Simultaneous $\mathrm{pH}$-recordings from multiple sites in children with and without distal gastroesophageal reflux. Journal of Pediatric Gastroenterology and Nutrition, 7, 46-51.

[5] Gustafsson, P. and Tibbling, L. (1988) 24-hour oesophageal two-level pH-monitoring in healthy children and adolescents. Scandinavian Journal of Gastroenterology, 23, 91-94.

[6] ESPGHAN-Working Group of the European Society of Pediatric Gastroenterology, Hepatology and Nutrition (1992) A standardized protocol for the methodology of esophageal $\mathrm{pH}$-monitoring and interpretation of data for the diagnosis of gastroesophageal reflux. Journal of Pediatric Gastroenterology and Nutrition, 14, 467-471.

[7] Ahrens, P., Haas, S. and Kitz, R. (2003) Standardization and optimization of 2-channel $\mathrm{pH}$-monitoring in children with gastroesophageal reflux-associated pulmonary disease. Monatsschrift Kinderheilkunde, 151, 1298-1305. doi:10.1007/s00112-003-0843-6

[8] Dobhan, R. and Castell, D.O. (1993) Normal and abnormal proximal esophageal acid exposure: Results of ambulatory dual-probe $\mathrm{pH}$-monitoring. The American Journal of Gastroenterology, 88, 25-29.

[9] Gorenstein, A., Levine, A., Boaz, M., Mandelberg, A. and Serour, F. (2003) Severity of acid gastroesophageal reflux assessed by $\mathrm{pH}$ metry: Is it associated with respiratory disease? Pediatric Pulmonology, 36, 330-334. doi:10.1002/ppul.10361

[10] Harding, S.M., Guzzo, M.R. and Richter, J.E. (1999) 24-h Esophageal pH-Testing in Asthmatics. Chest, 115, 
654-659. doi:10.1378/chest.115.3.654

[11] Jaspersen, D., Kulig, M., Labenz, J., Leodolter, A., Lind, T., et al. (2003) Prevalence of extra-oesophageal manifestations in gastro-oesophageal reflux disease: An analysis based on the ProGERD Study. Alimentary Pharmacology \& Therapeutics, 17, 1515-1520. doi:10.1046/j.1365-2036.2003.01606.x

[12] El-Serag, H.B., Gilger, M., Kuebeler, M. and Rabeneck, L. (2001) Extraesophageal association of gastroesophageal reflux disease in children without neurological defects. Gastroenterology, 121, 1294-1299. doi:10.1053/gast.2001.29545

[13] Coletti, R.B., Christie, D.L. and Orenstein, S.R. (1995) Indications for pediatric esophageal $\mathrm{pH}$-monitoring. Statement of the NASPGN. Journal of Pediatric Gastroenterology and Nutrition, 21, 253-262. doi:10.1097/00005176-199510000-00002

[14] Yüksel, H., Yilmaz, O., Kirmaz, C., Aydogldu, S. and Kasirga, E. (2006) Frequency of gastroesophageal reflux disease in nonatopic children with asthma-like airway disease. Respiratory Medicine, 100, 393-398.

[15] Arana, A., Bagucka, B., Hauser, B., Hegar, B. and Urbain, D., et al. (2001) pH-Monitoring in the distal and proximal esophagus in symptomatic infants. Journal of Pediatric Gastroenterology and Nutrition, 32, 259-264. doi:10.1097/00005176-200103000-00005
[16] Stordal, K., Johannesdottir, G.B., Bentsen, B.S., Knudsen, P.K. and Carlsen, K.C., et al. (2005) Acid suppression does not change respiratory symptoms in children with asthma and gastroesophageal reflux disease. Archives of Disease in Childhood, 90, 956-960. doi:10.1136/adc.2004.068890

[17] Vandenplas, Y., Goyvaerts, H. and Helven, R. (1991) Gastroesophageal reflux, as measured by 24-hour $\mathrm{pH}$-monitoring, in 509 healthy infants screened for risk of sudden infant death syndrome. Pediatrics, 88, 834-840.

[18] Mitchell, D.J., McClure, B.G. and Tubman, T.R. (2001) Simultanous monitoring of gastric and oesophageal $\mathrm{pH}$ reveals limitations of conventional oesophageal pH-monitoring in milk-fed infants. Archives of Disease in Childhood, 84, 273-276. doi:10.1136/adc.84.3.273

[19] Wenzel, T.G., Moroder, C., Trachterna, M., Thomson, M. and Silny, J., et al. (2002) Esophageal pH-monitoring and impedance measurement: A comparison of two diagnostic tests for gastroesophageal reflux. Journal of Pediatric Gastroenterology and Nutrition, 34, 519-523. doi:10.1097/00005176-200205000-00009

[20] Rosen, R., Lord, C. and Nurko, S. (2006) The sensitivity of multichannel intraluminal impedance and the $\mathrm{pH}$ probe in the evaluation of gastroesophageal reflux in children. Clinical Gastroenterology and Hepatology, 4, 167-172. doi:10.1016/S1542-3565(05)00854-2 